Thorax, 1979, 34, 749-754

\title{
Does sleep cause nocturnal asthma?
}

\author{
M R HETZEL AND T J H CLARK \\ From Brompton Hospital, London SW3, UK
}

ABSTRACT The effects of sleep interruption and deprivation were studied in 21 patients with nocturnal asthma. Seven patients were awakened at 0200 on three consecutive nights and exercised for 15 minutes. This produced no significant improvement in the overnight fall in peak expiratory flow rate (PEFR) compared with a control night of uninterrupted sleep.

In a second study in five patients PEFR was measured at two-hourly intervals to estimate the time of onset of the nocturnal fall in PEFR. On three subsequent nights they were awakened and exercised one hour before this time. This also failed to prevent a fall in PEFR by 0600 .

Eleven patients, who had followed a similar protocol to the second study, were kept awake until after 0300 or later, and PEFR was observed hourly. Six of them (group A) sustained their usual fall in PEFR while awake, proving that sleep was not responsible for their nocturnal asthma. Five patients (group B) showed little fall in PEFR until they were allowed to sleep, when an appreciable fall was noted on waking at 0600 . When sleep deprivation was repeated in two patients in group B, however, they sustained falls in PEFR while still awake.

We conclude that the circadian rhythm in PEFR is often in phase with the timing of sleep but sleep does not cause nocturnal asthma. Disruption of sleep therefore has no apparent value in the treatment of nocturnal asthma.

Nocturnal asthma has been recognised for centuries (Floyer, 1698). Regular monitoring of peak expiratory flow rate (PEFR) in asthma patients often shows a diurnal pattern with lowest readings at night (Turner-Warwick, 1977). Excessive diurnal variation may indicate an increased risk of sudden asthma death (Hetzel et al, 1977). Nocturnal asthma can be attributed to an exaggeration of a normal circadian rhythm in airway calibre (Reinberg and Gervais, 1972; Hetzel et al, 1978). Studies in asthmatic shift workers, however, (Clark and Hetzel, 1977) suggest a direct relation between sleep and the fall in PEFR on waking, irrespective of the time of day. We therefore studied the effects of sleep interruption and deprivation in asthma patients, both to test the hypothesis that sleep itself causes nocturnal asthma and to evaluate sleep derangement as a treatment for nocturnal asthma.

\section{Methods}

All patients gave informed consent to the studies that were carried out in hospital during convalescence from acute asthma. PEFR was routinely recorded with a Wright peak flow meter (Airmed) at four-hourly intervals, and always before drugs were given, from 0600-2200; three attempts were made on each occasion and the best used in analysis of results. All patients showed a PEFR pattern typical of nocturnal asthma (TurnerWarwick, 1977), with the lowest readings at 0600. They had a consistent fall in PEFR at 0600 of $>25 \%$ of the highest daily reading. Drug treatment was continued. Bronchodilator drugs were regularly spaced between 0600 and 2200 , usually at four-hourly intervals. Prednisone was given in divided doses between 0600 and 2200 or, in a few cases, as a single dose at 1000 . In all cases the last treatment was given at 2200 after PEFR had been measured. Treatment regimens were kept constant throughout the studies. Patients' times of waking and retiring to bed were strictly regulated to 0600 and 2200 hours respectively. Three protocols were followed.

Empirical waking-On the first control night, sleep was undisturbed from 2200 to 0600 . On three subsequent nights sleep was interrupted at 0200 and PEFR recorded. Patients left their beds and walked around the hospital corridors for $15 \mathrm{~min}$ utes. PEFR was then repeated, and they were left 
to sleep undisturbed until 0600. The falls in PEFR between 2200 and 0600 , measured as a percentage of the reading at 2200 , were compared with the control night.

Elective waking-On the first control night, sleep was undisturbed from 2200 to 0600 . On a second control night, patients were briefly awakened, without leaving their beds, to record PEFR at two-hourly intervals; sleep was otherwise undisturbed. From these two-hourly readings, the pattern of the overnight fall in PEFR was assessed for the approximate time of onset. On three subsequent nights patients were awakened one hour earlier than this time and then followed the same protocol as in study 1 . The percentage falls in PEFR on these three nights were compared with the control nights.

Elective waking and sleep deprivation-On the first three nights patients followed the same protocol as in study 2, except that they underwent only one night of sleep interruption and exercise. On the fourth night they were kept awake until one hour after the latest time that the nocturnal fall in PEFR had been observed on the previous three nights, including the timing of any spontaneous nocturnal attacks. They went to bed at 2200, adopted the sleeping posture but were kept awake with the lights on and spent their time reading or listening to the radio. PEFR was recorded every hour. When the appointed time had passed, they were allowed to sleep, with the lights off, until 0600 . The proportions of the overnight fall in PEFR that occurred while awake and asleep were compared.

After a rest period of at least four days, two patients repeated a night of sleep deprivation, staying awake until the same time as before. On this occasion they were not subjected to preceding nights of sleep interruption.

As patients were their own controls in all the experiments, Student's paired $t$ test was used to determine the significance of the difference between study and control nights for the overnight falls in PEFR.

\section{Results}

Twenty-one patients ( 7 men, 14 women) were recruited. Their mean age was 42 years (range 18-63). Twelve patients were atopic subjects with one or more positive $(>3 \mathrm{~mm})$ prick tests to common allergens. Mean duration of symptoms was 17 years (range 4-50). All patients were treated with sympathomimetic drugs by aerosol or by mouth, or both, and 18 also took sustained release preparations of salbutamol or aminophylline at 2200 . Seventeen patients received $\overline{\bar{c}}$ prednisone in doses of $15-40 \mathrm{mg}$ a day, and seven $\vec{\nabla}$ also used steroid aerosols. Two patients took sodium cromoglycate. Two patients participated in os studies 1 and 2 on separate admissions.

Empirical waking-Seven patients were studied. Table 1 compares the percentage falls in PEFR $\vec{\omega}$ from 2200 to 0600 on the control night and three $\overrightarrow{\vec{r}}$ nights of waking at 0200 . In one patient the nocturnal fall was abolished during sleep inter- $\oplus$ ruption and, as a result, the mean falls on the nights of sleep deprivation decreased. The other $\vec{c}$ patients, however, showed little consistent im-o provement, as in the example in fig 1 , and there? was no significant change in the mean results 0 from the control night. As seen in fig 1, the

Table 1 Empirical waking at $0200(n=7)$. Overnight fall in PEFR at 0600 as percentage of reading at 2200

\begin{tabular}{llrrr}
\hline Case & Control night & Night 1 & Night 2 & Night 3 \\
\hline 1 & 60.3 & 50.0 & 0.0 & 21.8 \\
2 & 51.2 & 0.0 & 0.0 & 0.0 \\
3 & 40.3 & 32.5 & $65 \cdot 3$ & 19.1 \\
4 & 44.4 & 51.2 & 19.7 & 2.9 \\
5 & 28.8 & 45.8 & 0.0 & 50.0 \\
6 & 43.0 & 21.3 & 35.1 & 50.0 \\
7 & 50.0 & 51.3 & 44.4 & 38.9 \\
\hline Mean & 45.4 & 36.0 & 23.5 & 26.1 \\
SEM & 3.7 & 7.3 & 9.7 & 7.8 \\
P & & 0.3 & $<0.1$ & $<.1$ \\
\hline
\end{tabular}

SEM $=$ Standard Error of Mean.

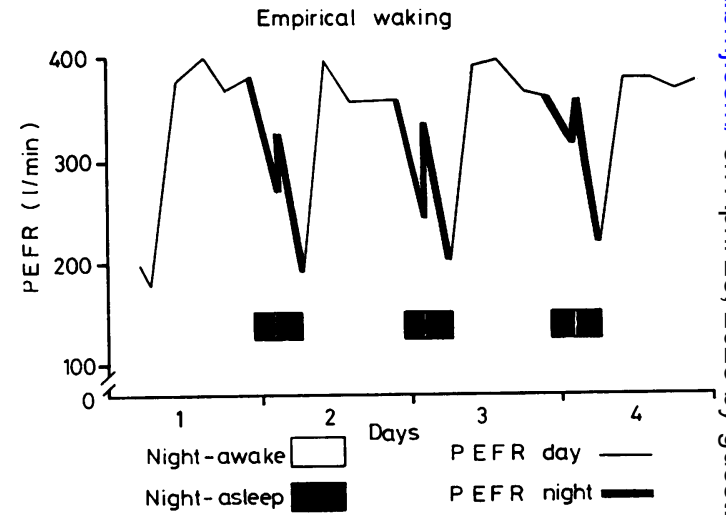

Fig 1 Effect of waking for 15 minutes' exercise on three consecutive nights at 0200. In this example a brief improvement is seen after exercise but a pronounced fall in PEFR occurs by 0600 with no improvement on control night results (not shown). Overnight rate of fall of PEFR, as determined by readings at 0200 , is variable. 
nocturnal fall in PEFR was often well developed by 0200 .

Elective waking-Five patients were recruited. There was no significant improvement in the nocturnal fall in PEFR on any of the three nights of sleep interruption (table 2). Although we had estimated the time of onset of the nocturnal fall in PEFR from two-hourly waking on the second control night, the rate of fall on the three subsequent nights was not sufficiently consistent to ensure that patients were always awakened before it had occurred (fig 2).

Elective waking and sleep deprivation-Eleven patients were studied. Their mean results are shown in table 3. Elective waking with 15 minutes' exercise was again ineffective in preventing the overnight fall in PEFR, and spontaneous nocturnal attacks occurred at 0300 after elective waking earlier in the night in two cases. Two distinct patterns of response to sleep deprivation were seen on the final night. Six patients (group A) sustained virtually all their nocturnal fall in PEFR while still awake. Five patients (group B) showed little or no fall while kept awake, but their usual fall in PEFR was observed on waking at 0600 after sleep for three hours or less. There was no difference in the mean duration of sleep deprivation in the two

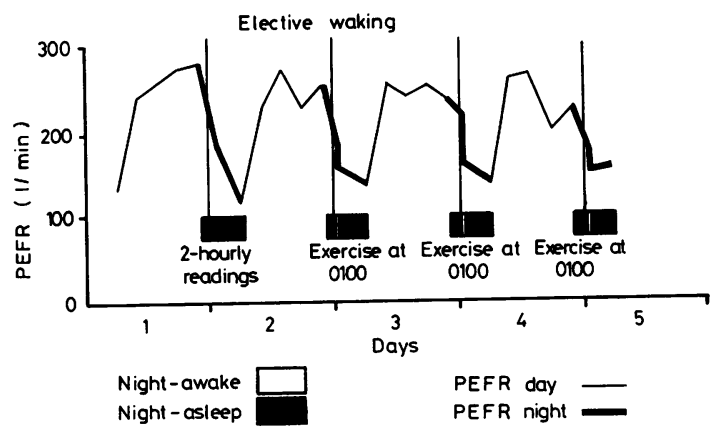

Fig 2 Elective waking. Time of onset of nocturnal fall in PEFR is estimated by two-hourly readings on a control night. Waking for exercise one hour before this time $(0100$ in this example) does not prevent overnight fall in PEFR, moreover, PEFR falls during exercise in case shown.

groups. No patient was observed to be asleep or remembered falling asleep at any time during sleep deprivation. Figures 3 and 4 show examples from groups A and B respectively. When a night of sleep deprivation was repeated in two patients in group $\mathrm{B}$, most of the nocturnal fall occurred while they were still awake.

Table 2 Elective waking $(n=5)$. Overnight fall in PEFR at 0600 as percentage of reading at 2200

\begin{tabular}{|c|c|c|c|c|c|}
\hline Case & Control night & Control night with 2-hourly PEFR & Night $1^{*}$ & Night $2^{*}$ & Night $3^{*}$ \\
\hline $\begin{array}{r}6 \\
7 \\
8 \\
9 \\
10\end{array}$ & $\begin{array}{l}42.5 \\
27.8 \\
15.4 \\
48.0 \\
54.5\end{array}$ & $\begin{array}{l}43 \cdot 5 \\
33 \cdot 3 \\
41 \cdot 5 \\
57 \cdot 1 \\
84 \cdot 8\end{array}$ & $\begin{array}{l}35 \cdot 7 \\
25 \cdot 0 \\
53 \cdot 7 \\
48 \cdot 0 \\
70 \cdot 0\end{array}$ & $\begin{array}{l}35 \cdot 7 \\
30 \cdot 7 \\
58 \cdot 6 \\
43 \cdot 5 \\
81 \cdot 8\end{array}$ & $\begin{array}{l}25 \cdot 8 \\
25 \cdot 0 \\
31 \cdot 4 \\
31 \cdot 1 \\
61 \cdot 1\end{array}$ \\
\hline $\begin{array}{l}\text { Mean } \\
\text { SEM } \\
\text { P }\end{array}$ & $\begin{array}{r}37 \cdot 6 \\
7 \cdot 1\end{array}$ & $\begin{array}{r}52.0 \\
9.0 \\
<\quad 0.1\end{array}$ & $\begin{array}{r}46.5 \\
7.7 \\
<0.4\end{array}$ & $\begin{array}{r}50.1 \\
9.2 \\
<0.3\end{array}$ & $\begin{array}{r}34.9 \\
6.7 \\
<0.7\end{array}$ \\
\hline
\end{tabular}

*Time of waking: $0100(n=1), 0200(n=3), 0400(n=1)$.

SEM = Standard Error of Mean.

Table 3 Elective waking and sleep deprivation $(n=11)$. Overnight fall in PEFR at 0600 as percentage of reading at 2200

\begin{tabular}{|c|c|c|c|c|c|c|}
\hline \multirow[b]{3}{*}{$\begin{array}{l}\text { Group A mean }(n=6) \\
\text { Group B mean }(n=5) \\
\text { Grand mean } \\
\text { SEM } \\
\text { P } \\
\text { Range }\end{array}$} & \multirow{3}{*}{$\begin{array}{l}\begin{array}{l}\text { Control } \\
\text { night }\end{array} \\
49 \cdot 2 \\
37 \cdot 8 \\
44 \cdot 0 \\
4 \cdot 0\end{array}$} & \multirow{3}{*}{$\begin{array}{l}\text { Control night with } \\
\text { 2-hourly PEFR } \\
34 \cdot 7 \\
37 \cdot 1 \\
35 \cdot 8 \\
5 \cdot 1 \\
<0 \cdot 3 \\
14-77\end{array}$} & \multirow{3}{*}{$\begin{array}{c}\begin{array}{c}\text { Elective } \\
\text { waking }\end{array} \\
42 \cdot 0 \\
44 \cdot 6 \\
43 \cdot 2 \\
4 \cdot 7 \\
<0 \cdot 9 \\
17-73\end{array}$} & \multicolumn{3}{|c|}{ Sleep deprivation } \\
\hline & & & & \multirow{2}{*}{$\begin{array}{l}\text { Pre-sleep } \\
36 \cdot 0 \\
3 \cdot 6 \\
21 \cdot 3\end{array}$} & \multicolumn{2}{|c|}{ Post-sleep ${ }^{*}$ Total } \\
\hline & & & & & $\begin{array}{r}2 \cdot 5 \\
45 \cdot 2 \\
21 \cdot 9 \\
\\
0-65\end{array}$ & $\begin{array}{c}38 \cdot 5 \\
48 \cdot 8 \\
43 \cdot 2 \\
5 \cdot 6 \\
<\quad 0 \cdot 9 \\
18-72\end{array}$ \\
\hline $\begin{array}{l}\text { Group B } \\
\text { Repeat study mean }(n=2)\end{array}$ & $41 \cdot 2$ & - & - & $24 \cdot 0$ & $4 \cdot 4$ & $28 \cdot 4$ \\
\hline
\end{tabular}

*Time of sleep onset: $0300(n=2), 0400(n=2), 0500(n=7)$.

$\mathrm{SEM}=$ Standard Error of Mean. 


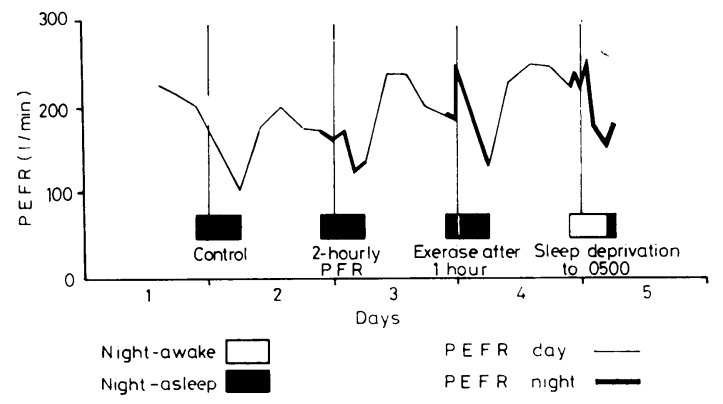

Fig 3 Again elective waking does not prevent overnight fall in PEFR, although a brief improvement is seen after exercise on third night. Nocturnal fall in PEFR develops during sleep deprivation to 0500 and cannot therefore be caused by sleep.

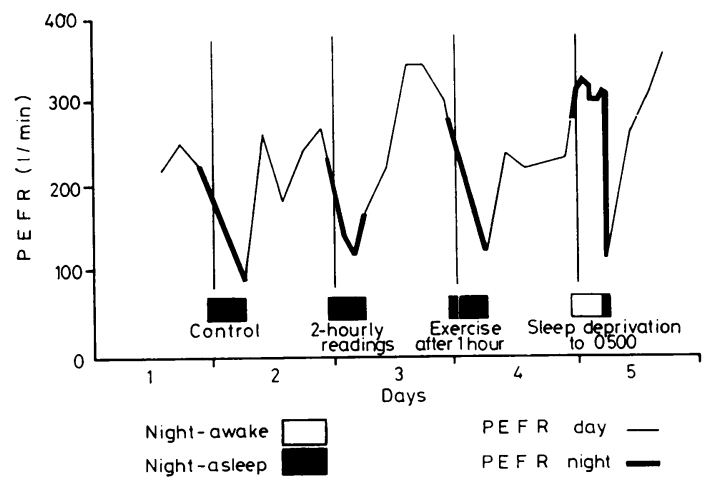

Fig 4 Elective waking on third night does not prevent overnight fall in PEFR and in this example $P E F R$ does not change after exercise. Sleep deprivation to 0500 is not associated with a fall in PEFR but a pronounced fall is seen after one hour's sleep from 0500 to 0600.

\section{Discussion}

In electroencephalographic studies Kales et al (1968) were unable to relate nocturnal asthma to any phase of sleep, although attacks were rare in the first hour of sleep. In asthmatic children, however (Kales et al, 1970), attacks did not occur during phase IV non-rapid eye movement sleep and in a nine-hour sleep period they were not observed in the first three hours when most of the phase IV sleep was seen. This implies that deep phases of sleep in the earlier part of the night may prevent nocturnal asthma. In keeping with this view we observed that in 315 nocturnal asthma attacks in another group of 15 asthma patients, only three attacks occurred in the first two hours of sleep, and the peak incidence occurred after six hours (unpublished data). Unfortunately, Kaleso et al $(1968,1970)$ did not measure respiratory을 function; thus patients in phase IV sleep may simply be too deeply unconscious to be awakened $\stackrel{\Phi}{\complement}$ by their airways obstruction. Moreover, the in-® cidence of phase IV sleep decreases with age $\vec{P}$ (Dement, 1960) but, in our experience, the? incidence of nocturnal asthma does not change.

Certain phases of sleep could, nevertheless, $\stackrel{?}{\rightleftharpoons}$ cause nocturnal asthma. As we had no facilities $\vec{x}$ for monitoring the electroencephalogram, we $\omega_{+}$ studied the effects of manipulation of sleep on the overnight fall in PEFR. We would have pre- $-\overrightarrow{ }$ ferred to discontinue treatment with drugs but this was considered unethical in patients with pronounced nocturnal asthma. The use and timing of $\vec{\square}$ drugs might affect the overnight fall in PEFR. $\mathbb{D}$ Patients recruited to this study, however, showed $\Phi$ pronounced nocturnal asthma despite treatment. $\frac{3}{0}$ As the timing and dosage of drugs was kept constant during the study, it seems unlikely that the $\vec{\varphi}$ drugs used had any important effect on the results. $\widehat{ }$ Observations on the effects of sleep deprivation depend on patients having stayed awake during this period, and ideally one would monitor the electroencephalogram to prove this. Although we lacked this facility and simply observing patients $\frac{\circ}{\mathbb{D}}$ may possibly not detect brief periods of sleep, we think it unlikely that patients slept to any appreciable degree during sleep deprivation.

The question of which daytime reading to compare with the subsequent 0600 result to measure the degree of nocturnal asthma needs to be considered. As the reading at 2200 was im- $\underset{x}{x}$ mediately related to the onset of sleep and pre-i ceded the last treatment of the day, we thought it $\underline{3}$. was the most logical choice. As can be seen from 8 the figures, however, the highest daily readings occurred between 1400 and 2200, in keeping with 윽 our previous studies of the phase of the PEFR $D$ rhythm in larger numbers of asthmatic and normal o subjects (Hetzel et al, 1978), and in some patients the timing of the highest reading varied from day or to day. Nevertheless, when the reading at $2200 \mathrm{~N}$ was not the highest, it was representative of it. N

Study 1 represented the simplest method of sleep interruption but was effective only in one patiento whose daytime asthma was also obviously improv- $\mathbb{\Phi}$ ing. Having often observed in study 1 that the fall $\stackrel{?}{+}$ in PEFR was well established by 0200 , we tried to $\frac{0}{0}$ anticipate its onset in study 2 by examining the pattern of the nocturnal fall with two-hourly $\stackrel{\square}{\Phi}$ PEFR readings on a second control night. Briefly $\underset{\perp}{\varrho}$ waking patients in this way should not disturb응 sleep cycles. Dement (1960) and Kales et al (1964) 
have shown that waking for a few minutes has no effect on the pattern of sleep cycles unless repeatedly performed at the onset of every rapid eye movement sleep; the dream deprivation phenomenon may then result with increasingly frequent rapid eye movement periods. This effect is not seen in a single night. Unfortunately, elective waking before the anticipated fall in PEFR was no more effective in preventing nocturnal asthma than empirical waking at 0200 .

Study 3 produced two distinct responses. In group $A$ the nocturnal attack occurred in the absence of sleep on the night of sleep deprivation and could not, therefore, be caused by it. Group B patients seemed dependent on sleep for the nocturnal attack to occur and one hour of sleep was sometimes sufficient (fig 4). Attempts to demonstrate this effect again in two patients were, however, unsuccessful. We therefore suspect that this apparent causal relation between sleep and the nocturnal fall in PEFR was coincidental; as seen in study 2, the rate of overnight fall in PEFR varied from night to night in some patients.

In interpreting these observations it is important to remember that sleep forms part of a circadian rhythm. Free-running experiments in bunkers with constant light or darkness show a natural rhythm in man of some eight hours' sleep in a circadian period of 24-27 hours (Aschoff, 1965; Pöpel, 1968). In normal life light and other synchronisers regulate the biological clock to the 24-hour period of the solar day. Artificially increasing the duration of light in a human subject's day will rapidly alter the phase of his sleep-wakefulness rhythm within the next 24 hours (Aschoff, 1967). Othmer and Heyden (1974) have shown that rapid eye movement periods in the electroencephalogram occur in both the sleeping and waking states and show circadian periodicity. Thus sleep, the electroencephalogram, and PEFR all show circadian features, and any apparent causal relation between sleep and the nocturnal fall in PEFR might be simply accounted for by these two rhythms having a similar phase. Low peak flow rates tend to coincide with the period of sleep, but the PEFR and sleep-wakefulness rhythms may be quite independent of each other or both subservient to another, master, biological clock. A cause and effect relation between two rhythms can sometimes be disproved by exposing subjects to a change of shift; if one rhythm alters phase more rapidly than the other they may become separable for a short time. In asthmatic shift workers, however, we were unable to separate the PEFR rhythm from periods of sleep and the subjects available all rapidly changed the phase of their PEFR rhythms on starting a new shift (Clark and Hetzel, 1977).

The initial impression in patients in group $B$ that a brief period of sleep at the end of the night can cause nocturnal asthma may possibly be accounted for as the result of sleep interruption on the previous nights. The exposure to light necessitated by waking patients on these earlier nights may have progressively delayed the phase of the PEFR rhythm so that its bathyphase (lowest point) coincided with the short period of sleep permitted on the night of sleep deprivation. Two subjects who repeated the night of sleep deprivation were not subjected to these preliminary nights of sleep interruption again. This may have allowed the PEFR rhythm to revert to its normal phase so that its bathyphase was now seen while patients were still awake. Pittendrigh (1965) has shown in plants and animals that brief periods of exposure to light during the night can alter the phase of their rhythms on subsequent nights. Exposure in the first half of the night advances the phase while light in the later half delays it; thus organisms progressively adapt to seasonal changes in the times of dawn and dusk. In free-running experiments in constant light or darkness these advances and delay effects balance to produce the natural circadian period that usually has a cycle slightly longer than 24 hours.

In conclusion we make no claim to have excluded any causal relation between sleep and nocturnal asthma as the relation between them is evidently complex. It is difficult to conceive of experiments that could guarantee to alter the time of sleep without affecting the phase of the PEFR rhythm as well. From a clinical viewpoint, however, these studies offer little hope that sleep disruption would have any value as a treatment for nocturnal asthma. Severely disabled patients might, however, benefit from waking to take further drugs during the night. The only chronobiological technique that we have found potentially beneficial is the rapid scrambling of patients' PEFR rhythms; as seen in some asthmatic shift workers whom we have more recently studied, very frequent changes of shift reduce the amplitude of the rhythm (Hetzel and Clark, 1978). Few patients, however, would find this treatment socially acceptable.

-We thank the physicians of Brompton Hospital for permission to study patients under their care. The study was supported by a grant from the Board of Governors, Brompton Hospital. 


\section{References}

Aschoff, J (1965). Circadian rhythms in man. Science, 148, 1427-1432.

Aschoff, J (1967). In Life Sciences and Space Research, edited by $\mathrm{H}$ Brown and F Favorite, pp 159. 173. North Holland, Amsterdam.

Clark, T J H, and Hetzel, M R (1977). Diurnal variation of asthma. British Journal of Diseases of the Chest, 71, 87-92.

Dement, W (1960). The effect of dream deprivation. Science, 131, 1705-1707.

Floyer, J (1698). A Treatise of the Asthma, R Wilkin and $W$ Innys, pp 7-8. London.

Hetzel, M R, Clark, T J H, and Branthwaite, M A (1977). Asthma: analysis of sudden deaths and ventilatory arrests in hospital.. British Medical Journal, 1, 808-811.

Hetzel, M R, Clark, T J H, and Brown, D (1978). Normal circadian rhythms in peak expiratory flow rate. Thorax, 33, 668 .

Hetzel, M R, and Clark, T J H (1978). The clinical importance of circadian factors in severe asthma. Chronobiologia, 5, 196-197.

Kales, A, Hoedemaker, F S, Jacobson A, and Lichtenstein, E L (1964). Dream deprivation: an experimental reappraisal. Nature, 204, 1337-1338.

Kales, A, Beall, G N, Bajor, G F, Jacobson, A, and Kales, J D (1968). Sleep studies in asthmatic adults: relationship of attacks to sleep stage and time of night. Journal of Allergy, 41, 164-173.

Kales, A, Kales, J D, Sly, R M, Scharf, M B, Tan, $\frac{\bar{w}}{2}$ $T$ L, and Preston, T A (1970). Sleep patterns of $\mathbb{Q}$ asthmatic children. All night electroencephalographic studies. Journal of Allergy, 46, 300-308.

Othmer, E, and Hayden, M (1974). Rapid eye move- $\vec{O}$ ments during sleep and wakefulness. In Chronobiology, edited by $\mathrm{L}$ Scheving, F Halberg, and J $\vec{\omega}$ Pauling, pp 491-495. Igaku Shoin, Tokyo.

Pittendrigh, C S (1965). In Circadian Clocks, edited by J Aschoff, pp 277-297. North Holland, $\underset{\perp}{\omega}$ Amsterdam.

Pöppel, E (1968). Desynchronisationen circadianerRhythmen innerhalb einer isolierten Gruppe. $\overrightarrow{0}$ Pfluegers Arch Gesamte Physiol Menschen Tiere, 음 299, 364-370.

Reinberg, A, and Gervais, $P$ (1972). Circadian rhythms in respiratory functions with special ${ }_{\infty}^{\mathbb{D}}$ reference to human chronophysiology and chrono- $\stackrel{\oplus}{\longrightarrow}$ pharmacology. Bulletin de Physiopathologie Respiratoire, 8, 663-675.

Turner-Warwick, M (1977). On observing patterns of $\vec{\varphi}$ airflow obstruction in chronic asthma. British Journal of Diseases of the Chest, 71, 73-85.

Requests for reprints to: $\operatorname{Dr}$ M R Hetzel, Brompton Hospital, Fulham Road, London SW3 6HP.

\section{Second International Symposium on Psychopathological and Neurological Dysfunctions following Open Heart Surgery}

Milwaukee, Wisconsin, USA: 6-7 March 1980

Further information from: Jeremy $\mathbf{M}$ Katz, PhD, American Symposium Director, Second International Symposium, Department of Psychiatry-The Medical College of Wisconsin, Milwaukee County Mental Health Center, 9191 Watertown Plank Road, Milwaukee, Wisconsin 53226, USA. 